\title{
Functionalization of Single-Wall Carbon Nanotubes: An Assessment of Computational Methods
}

\author{
Brahim Akdim ${ }^{1}$, Tapas $\mathrm{Kar}^{2}$, Xiaofeng Duan ${ }^{1}$, and Ruth Pachter ${ }^{1}$ \\ ${ }^{1}$ Air Force Research Laboratory, Materials and Manufacturing Directorate \\ Wright-Patterson Air Force Base, OH, USA \\ \{Brahim.Akdim, Xiaofeng.Duan, Ruth.Pachter\} @wpafb.af.mil \\ ${ }^{2}$ Department of Chemistry and Biochemistry, Utah State University, Logan, UT, USA \\ tkar@wpafb.af.mil
}

\begin{abstract}
We summarize a theoretical study for modeling functionalization of single-wall carbon nanotubes (SWCNTs), specifically first principles density functional theory calculations, as compared to semi-empirical or simplified hierarchical methods. We focus on the assessment of the methods to be applied to obtain reliable results and gain a fundamental understanding of the diazotization and ozonolysis of SWCNTs. Computational challenges encountered are highlighted.
\end{abstract}

\section{Introduction}

Applications of SWCNTs are still limited by the inability to carefully control the behavior of these materials, for example, with respect to the separation of metallic vs. semiconducting tubes, or nanotubes with different diameters. Thus, a number of chemical functionalization and solubilization studies emerged, recently reviewed [1], including direct attachments of functional groups to the side-wall of a SWCNT using diazonium reagents [2,3,4], solvent free functionalization [5], fluorination and subsequent derivatization [6], or functionalization by strong oxidizing agents, exploring various oxidants [7], due to the inherent strain in SWCNTs, rationalized, in part, by the pyramidalization angle [8]. Most recently, structure-based sorting by sequencedependent DNA assembly was reported [9]. In this study, we examined the diazotization [10], and ozonolysis, which was shown to enhance solubility [11], of SWCNTs.

In order to gain insight into the functionalization mechanisms of SWCNTs theoretically, a large number of atoms have to be included in quantum mechanical calculations, which could become infeasible [12]. Hence, mixed QM/MM methods, such as ONIOM, introduced by Morokuma et al. [13,14,15,16], were found appropriate [17] for modeling large molecular/nano systems. Within the ONIOM scheme, a first principles calculation is performed on a small part of the system, while the remaining atoms are treated at a lower level of theory, such as by semi-empirical or empirical methods. 
In the two-layered scheme, $\mathrm{E}_{\mathrm{ONIOM}}$ is given by:

$$
E_{\text {ONIOM }}=E_{3}+E_{2}-E_{1}
$$

where $\mathrm{E}_{3}$ and $\mathrm{E}_{1}$ relate to the low-level theory of the complete and model systems, respectively, while $\mathrm{E}_{2}$ is the energy of the model system computed at a high-level of theory. The multi-layer ONIOM method has been formulated [18]. However, for an accurate calculation, the system partitioning into subunits has to be applied with care. For example, in modeling nitrogen atoms interacting with carbon clusters, including a $(9,0)$ nanotube [19], and using a range of sizes for the high-level portion, a large disparity in the binding energies was calculated, ranging from -16.5 to -78.9 $\mathrm{kcal} / \mathrm{mol}$, for the doublet state, depending on the model used. In our investigation, we examine the reliability of ONIOM for modeling SWCNT functionalization.

\section{Computational Details}

$\mathrm{C}(5,5)$ SWCNTs were used in all calculations, where 2-phenylimidazole (L) was modeled, functionalized at the SWCNT. The binding energy (BE) was calculated as follows:

$$
B E=E(L-S W C N T)-[E(S W C N T)+E(L)]
$$

A positive value of $\mathrm{BE}$ indicates an endothermic reaction, whereas the exothermic reaction is associated with a negative $\mathrm{BE}$. Note that the adjacent carbon to the functional group is saturated with a hydrogen atom (cf. Figure 1a).

ONIOM calculations [20], applying B3LYP/6-31G*, were carried out with varying sizes of high-level SWCNT models, of 2, 12, and 16 carbon-atoms, while the full system was treated with a semi-empirical, or an empirical UFF (Universal Force Field) method [21]. The functionalized SWCNT was also modeled from first principles by using the B3LYP exchange-correlation functional with $3-21 \mathrm{G}$ and $6-31 \mathrm{G}^{*}$ basis sets, and with varying tube lengths to assess system size effects, ranging from 5 (Figure 1b) to 11 unit cells. We note that our largest calculation (11 SWCNT unit cells, and the functional unit, at the B3LYP/3-21G level) consisted of 279 atoms. These calculations were carried out on a SGI Origin 3900, using 8 processors (300 $\mathrm{MW}$ in memory/CPU). A single SCF iteration's timing was ca. 5 minutes/CPU, and about 20 SCF iterations for an optimization cycle were required.

Simulations applying periodic boundary conditions were carried out using an allelectron linear combination of atomic orbitals DFT approach [22], previously shown to be appropriate for modeling nanotubes [23]. The Perdew-Burke-Ernzerhof (PBE) exchange-correlation functional [24], within the generalized gradient approximation, was applied, to reduce the over-binding effects caused by the local density approximation, and known to adequately predict the density of states as compared to experiment [25]. A double numerical d-functions basis set was used. To avoid intertube interactions, a unit cell with a distance of $30 \AA$ in the direction perpendicular to the 
tube axis, separating a tube and its image, was employed. In the direction of the tube axis, 3 and 5 SWCNT units were studied (Figures 1c and 1d, respectively).

(a)

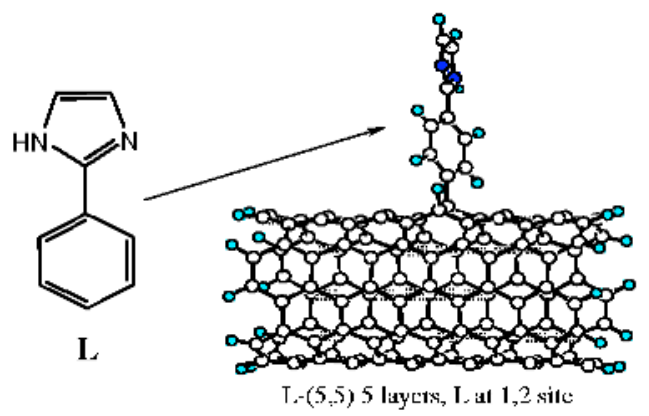

(b)<smiles>COC1(C)OC(C)C(C)OC1OB1OC(C)=C(C)C(C)O1</smiles>

(c)

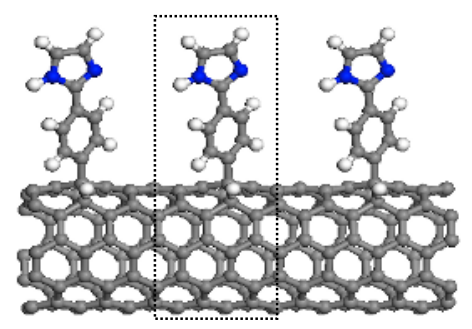

(d)

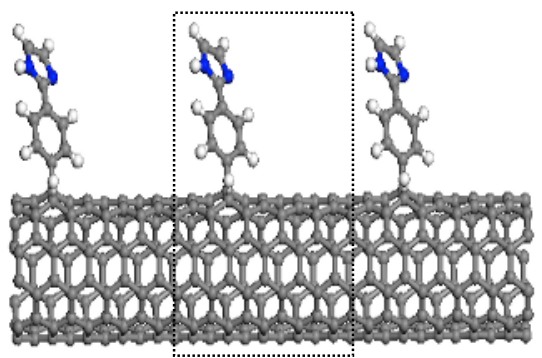

Fig. 1. Atomistic systems studied: (a) L-functionalized 5-unit cell SWCNT; (b) numbering for L; (c) and (d): 3- and 5- SWCNT units with periodic boundary conditions; the box delimits the super-cell used in the simulation 


\section{Results and Discussion}

\subsection{2-Phenylimidazole}

The optimized 2-phenylimidazole structures show significant differences between the semi-empirical and DFT/B3LYP exchange-correlation functional results (e.g., bond lengths reported in Table 1; numbering in Fig. 1b). Moreover, notable differences were obtained for the inter-ring torsional angle, or co-planarity, namely, $11.2 \mathrm{deg}, 30$ $\mathrm{deg}$, and $90 \mathrm{deg}$, when applying B3LYP/6-31+G**, PBE/DNP, or semi-empirical MNDO and PM3, respectively. Interestingly, the PBE/DNP result is in better agreement with previous Hartree-Force/6-31G** calculations for the ground state of 2phenylimidazole of ca. $19 \mathrm{deg}[26]$ than the B3LYP/6-31+G** result. A co-planar configuration was noted for the excited state [26].

Table 1. Optimized structure of 2-phenylimidazole; values in $\AA$

\begin{tabular}{llllllll}
\hline Bonds & MNDO & AM1 & PM3 & PM5 & $3-21 \mathrm{G}^{\mathrm{a}}$ & $6-31 \mathrm{G}^{* \mathrm{a}}$ & $6-31+\mathrm{G}^{*} *^{\mathrm{a}}$ \\
\hline $1-3$ & 1.404 & 1.394 & 1.391 & 1.387 & 1.399 & 1.398 & 1.399 \\
$3-4$ & 1.406 & 1.393 & 1.390 & 1.386 & 1.391 & 1.391 & 1.393 \\
$1-7$ & 1.405 & 1.394 & 1.391 & 1.387 & 1.396 & 1.395 & 1.397 \\
$4-10$ & 1.419 & 1.404 & 1.400 & 1.396 & 1.406 & 1.406 & 1.407 \\
$9-10$ & 1.419 & 1.403 & 1.400 & 1.395 & 1.404 & 1.404 & 1.406 \\
$9-7$ & 1.405 & 1.394 & 1.389 & 1.386 & 1.395 & 1.394 & 1.396 \\
$10-11$ & 1.470 & 1.462 & 1.457 & 1.457 & 1.459 & 1.466 & 1.467 \\
$2-11$ & 1.352 & 1.366 & 1.360 & 1.355 & 1.337 & 1.326 & 1.326 \\
$5-11$ & 1.401 & 1.410 & 1.399 & 1.400 & 1.385 & 1.374 & 1.375 \\
$6-2$ & 1.387 & 1.387 & 1.387 & 1.397 & 1.393 & 1.371 & 1.372 \\
$8-5$ & 1.397 & 1.390 & 1.399 & 1.403 & 1.393 & 1.378 & 1.379 \\
$8-6$ & 1.394 & 1.409 & 1.387 & 1.385 & 1.376 & 1.374 & 1.376 \\
\hline
\end{tabular}

${ }^{\mathrm{a}} \mathrm{B} 3 \mathrm{LYP}$ exchange-correlation functional.

\subsection{Diazotization}

Table 2 summarizes the binding energies for a $\mathrm{C}(5,5)$ functionalized SWCNT (Fig. 1a). In order to ascertain the applicability of our calculations to model functionalization of SWCNTS, which, as was pointed out, are known to require special conditions for reactions to take place, no recourse as to a suggested mechanism was taken at this stage. The results show the reaction to be endothermic, also when periodic boundary conditions were adopted, where BEs of $40 \mathrm{kcal} / \mathrm{mol}$ and $43 \mathrm{kcal} / \mathrm{mol}$ for the 3 - and 5 unit cell SWCNT models, respectively, were calculated. We note that the closest distances between an atom in the functional group and its image in these models are in the order of $4.3 \AA$ and $8.9 \AA$, respectively, as shown in Figs. 1c-d.

In probing the effects of different unit-cell sizes without periodic boundary conditions, we find, once again, the reaction to be endothermic, with a BE of ca. 
40kcal/mol, for 5- and 7- unit cells, respectively. A lower value has been obtained for the 11-unit cell (Table 2), possibly due to the smaller basis set applied. These results further confirm that such reactions are difficult to occur.

Table 2. Binding energies (BE, $\mathrm{kcal} / \mathrm{mol}$ ) obtained with different theoretical methods

\begin{tabular}{ccc}
\hline & Methods & BE $(\mathrm{kcal} / \mathrm{mol})$ \\
\hline 5- unit-cell & B3LYP/6-31G* & 39.68 \\
& AM1 & 17.02 \\
MNDO & 7.83 \\
PM3 & 38.21 \\
PM5 & 3.10 \\
& ONIOM(B3LYP/6-31G*:UFF) & $-43.59^{\mathrm{a}}$ \\
& (2 Carbon model) & $-20.78^{\mathrm{b}}$ \\
& ONIOM(B3LYP/6-31G*:UFF) & $29.66^{\mathrm{a}}$ \\
& (12 Carbon model) & $-97.63^{\mathrm{b}}$ \\
ONIOM(B3LYP/6-31G*:UFF) & $-7.90^{\mathrm{a}}$ \\
11- unit-cell & (16 Carbon model) & $35.85^{\mathrm{b}}$ \\
\hline
\end{tabular}

binding energy using ${ }^{\mathrm{a} O N I O M}$ extrapolated energy;

${ }^{b}$ high level energy part of the pertinent model.

Calculations with ONIOM provided varying results, depending on the model size, emphasizing the importance of applying high-level first-principles methods (Table 2). These disparities may invalidate the application of this approach for modeling reliably the functionalization of SWCNTs. However, when modeling functionalization of SWCNTs with terminal carboxylic groups, obtained by oxidation [27, 28], where less subtle changes occur, the results are less sensitive to the use of ONIOM.

\subsection{Ozonolysis}

Ozonolysis was previously investigated by applying ONIOM [29], indicating that the 1,3 cycloaddition of $\mathrm{O}_{3}$ onto the sidewall of a $\mathrm{C}(5,5)$ tube is possible and a binding energy of $-38.7 \mathrm{kcal} / \mathrm{mol}$ has been estimated using B3LYP/6-31G*:AM1. In another study, Lu et al. [30] found a binding energy of $-31.3 \mathrm{kcal} / \mathrm{mol}$, considering a 6-layer tube, while first principles calculations were also performed [31]. Most recently, sidewall epoxidation of SWCNTs was studied with ONIOM [32]. In our study, 2and 16-carbon atoms ONIOM calculations were performed within the two-layered scheme (Fig. 2). The (B3LYP/6-31G*:AM1) results are consistent with previous work. More accurate calculations, such as (B3LYP/6-31G*//B3LYP/6-31G*), result in different binding energies (Table 3).

The discrepancies when applying a 2- vs. 16-atom model within ONIOM emphasize, once again, the importance of an appropriate partitioning of the molecular 
model. Furthermore, within the same model, different results were obtained when changing the low-level of theory (UFF or AM1), with an estimated difference of about $18 \mathrm{kcal} / \mathrm{mol}$.

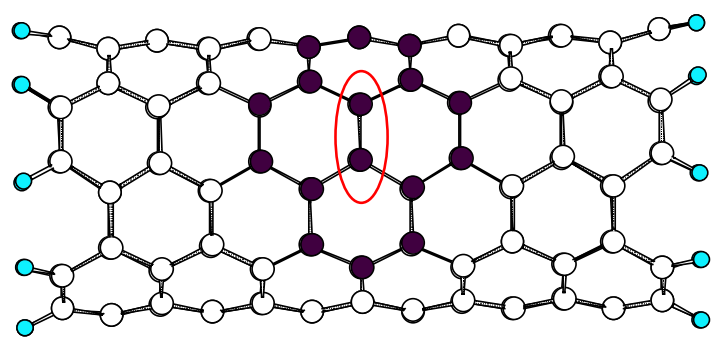

Fig. 2. ONIOM models for modeling ozonolysis: the oval circle points to the 2- carbon models, whereas the filled circles are the 16- carbon models.

Table 3. Ozonolysis of SWCNTs for different levels of theory

\begin{tabular}{cc}
\hline & \\
Methods & BE(kcal/mol) \\
\hline AM1 & -0.26 \\
B3LYP/3-21G//B3LYP/3-21G & -48.70 \\
B3LYP/6-31G*//B3LYP/3-21G & -23.51 \\
B3LYP/6-31G*//B3LYP/6-31G* & -19.66 \\
ONIOM B3LYP/6-31G*:UFF & $-34.32^{\mathrm{a}}$ \\
(2-carbon model) & $-59.6^{\mathrm{b}}$ \\
ONIOM B3LYP/6-31G*:AM1 & $-10.16^{\mathrm{a}}$ \\
(2-carbon model) & $-72.91^{\mathrm{b}}$ \\
ONIOM B3LYP/6-31G*:UFF & $-13.40^{\mathrm{a}}$ \\
(16-carbon model) & $-7.17^{\mathrm{b}}$ \\
ONIOM B3LYP/6-31G*:AM1 & $-31.34^{\mathrm{a}}$ \\
(16-carbon model) & $-15.64^{\mathrm{b}}$ \\
\hline
\end{tabular}

binding energy using aNIOM extrapolated energy;

bhigh level part of the pertaining model.

\section{Conclusions}

Overall, as anticipated, our calculations show the diazotization of SWCNTs to be endothermic, while ozonolysis is exothermic. In assessing an appropriate level of theory to be applied in modeling functionalization of SWCNTs, we find that density functional theory calculations are preferred, while although the ONIOM model with a large number of atoms at a high-level of theory could provide reliable energetics, care 
should be taken in defining a suitable model size within this framework. Indeed, to understand the proposed reaction mechanisms with SWCNTs, where water-soluble diazonium salts exhibit highly chemoselective reactions with metallic vs. semiconducting tubes, we currently apply DFT to calculate the electronic structures with respect to the reaction paths [33].

\section{References}

1. Sun, Y.-P., Fu, K., Lin, Y., Huang, W., Acc. Chem. Res. 35 (2002) 1096

2. Bahr, J. L., Tour, J. M., Chem. Mater. 13 (2001) 3823

3. Bahr, J. L., Yang, J., Kosynkin, D. V., Bronikowski, M. J., Smalley, R. E., Tour, J. M., J. Am. Chem. Soc. 123 (2001) 6536

4. Strano, M. S., Dyke, C. A., Usrey, M. L., Barone, P. W., Allen, M. J., Shan, H., Kittrell, C., Hauge, R. H., Tour, J. M., Smalley, R. E., Science 301 (2003) 1519

5. Dyke, A., Tour, J. M., J. Am. Chem. Soc. 125 (2003) 1156

6. Khabashesku, V. N., Billups, W. E., Margrave, J. L., Acc. Chem. Res. 35 (2002) 1087

7. Zhang, J., Zou, H., Qing, Q., Yang, Y., Li, Q., Liu, Z., Guo, X., Du, Z., J. Phys. Chem. B 107 (2003) 3712

8. Niyogi, S., Hamon, M. A., Hu, H., Zhao, B., Bhowmik, P., Sen, R., Itkis, M. E., Haddon, R. C., Acc. Chem. Res. 35 (2002) 1105

9. Zheng, M., Jagota, A., Strano, M, S., Santos, A. P., Barone, P., Chou, S. G., Diner, B. A., Dresselhaus, M, S., Mclean, R, S., Onoa, G. B., Samsonidze, G. G., Semke, E. D., Usrey, M., Walls, D. J., Science 302 (2003) 1545

10. Dang, T., Vaia, R. private communication.

11. Cai, L., Bahr, J. L., Yao, Y., Tour, J. M., Chem. Mater. 14 (2002) 4235

12. Schmidt, M. W., Baldridge, K. K, Boatz, J. A., Elbert, S. T., Gordon, M. S., Jensen, J. H., Koseki, S., Matsunaga, N., Nguyen, K. A., Su, S., Windus, T. L., Dupuis, M., Montgomery, J. A., J. Comput. Chem., 14 (1993) 1347

13. Maseras, F., Morokuma, K., J. Comput. Chem. 16 (1995) 1170

14. Humbel, S., Sieber, S. S., Morokuma, K., J. Chem. Phys. 105 (1996) 1959

15. Dapprich, S., Komáromi, I., Byun, K. S., Morokuma, K., Frisch, M. J., J. Mol. Struct. Theochem 462 (1999) 1

16. Vreven, T., Morokuma, K., J. Comput. Chem. 21 (2000) 1419

17. Vreven T., Morokuma K., Farkas O., Schlegel H. B. Frisch M. J., Cherry L. J Comput. Chem. 24 (2003) 760

18. Tschumper, G. S., Morokuma, K., J. Mol. Struct. Theochem 592 (2002) 137

19. Walch, S. P., Chem. Phys. Lett. 374, (2003) 501

20. Gaussian2003,_http://www.gaussian.com/

21. Rappe, A. K., Casewit, S. J., Goddard, W. A., Skiff, W. M., J. Am. Chem. Soc., 114 (1992) 10024

22. Delley, B. J., Chem. Phys. 113 (2000) 7756; implemented by Accelyrs, Inc.

23. Akdim, B., Duan, X., Adams, W. W., Pachter, R., Phys. Rev. B. 67 (2003) 245404

24. Perdew, J. P., Burke, K., Ernzerhof, M., Phys. Rev. Lett. 77 (1996) 3865

25. Avramov, P. V., Kudin, K. N., Scuseria, G. E., Chem. Phys. Lett. 370 (2003) 597

26. Catalan, J., de Paz, J. L. G., del Valle, C., J., Kasha, M., J., Phys. Chem. A 1015284 (1997)

27. Basiuk, V. A., Basiuk, E. V., Saniger-Blesa, J-M., Nano Lett., (2001) 657 
28. Basiuk, V. A., Nano Lett., (2002) 835

29. Lu, X., Zhang, L., Xu, X., Wang, N., Zhang, Q., J. Phys. Chem. B 106 (2002) 2136

30. Lu, X., Tian, F., Xu, X., Wang, N., Zhang, Q., J. Am. Chem. Soc. 125 (2003) 7923

31. Duan, X., Akdim, B., Pachter, R., Dekker Encyclopedia of Nanoscience and Nanotechnology, in press.

32. Lu, X., Qinghong, Y., Zhang, Q., Org. Lett. 5 (2003) 3527

33. Duan, X., Akdim, B., Pachter, R., work in progress. 